\title{
Frozen Plasma Within the Flow From a Metal Plate Hit by an Electron Beam Pulse
}

\author{
M. Garcia
}

November 12, 1997

This is an informal report intended primarily for internal or limited external distribution. The opinions and conclusions stated are those of the author and may or may not be those of the Laboratory.

Work performed under the auspices of the U.S. Department of Energy by the Lawrence Livermore National Laboratory under Contract W-7405-Eng-48. 


\section{DISCLAIMER}

This document was prepared as an account of work sponsored by an agency of the United States Government. Neither the United States Government nor the University of California nor any of their employees, makes any warranty, express or implied, or assumes any legal liability or responsibility for the accuracy, completeness, or usefulness of any information, apparatus, product, or process disclosed, or represents that its use would not infringe privately owned rights. Reference herein to any specific commercial product, process, or service by trade name, trademark, manufacturer, or otherwise, does not necessarily constitute or imply its endorsement, recommendation, or favoring by the United States Government or the University of California. The views and opinions of authors expressed herein do not necessarily state or reflect those of the United States Government or the University of California, and shall not be used for advertising or product endorsement purposes.

This report has been reproduced directly from the best available copy.

Available to DOE and DOE contractors from the Office of Scientific and Technical Information P.O. Box 62, Oak Ridge, TN 37831

Prices available from (615) 576-8401, FTS 626-8401

Available to the public from the National Technical Information Service

U.S. Department of Commerce 5285 Port Royal Rd. Springfield, VA 22161 


\title{
Frozen plasma within the flow from a metal plate hit by an electron beam pulse
}

\author{
Manuel Garcia \\ 12 November 1997 \\ Lawrence Livermore National Laboratory, L-153, POB 808, Livermore, CA 94550 \\ garcia22@1lnl.gov, (510) 422-6017, FAX (510) 423-5080
}

When a pulsed electron beam hits a metal plate with sufficient energy a volume of the metal becomes hot fluid that subsequently sprays out of the plate. A portion of this flow is ionized. This report describes a fluid that expands so rapidly into a vacuum that the ionized portion of the flow departs from local thermodynamic equilibrium. This cold supersonic exhaust will have a much higher degree of ionization, and of higher electron temperature, than would be expected from a gas in thermodynamic equilibrium at the local temperature of the neutral flow. This report presents a continuation of the work described in reference (1).

My aim is to develop as simple a model as will reasonably predict the speed and density of neutral flow, and the temperature and density of plasma. I use simplifying assumptions and mathematical approximations to develop convenient formulas, and I test them by comparing specific examples to experimental data and computations by DeVolder and other Los Alamos scientists (2).

The phenomenon of sudden expansion of a gas cloud into vacuum is described in several sections of the two-volume work by Zel'dovich and Raizer (3). The criterion I use for estimating the point in the flow where plasma ceases to be in equilibrium is in principle that proposed by Bray (4), (5), and discussed extensively by Vincenti and Kruger (6).

The immediate concern motivating this work is how to design a metal target that accurately converts an electron beam pulse train into a radiation pulse train for use in the high-speed radiography of implosion hydrodynamics experiments. In the radiography 
application, either the electron beam must be swept magnetically along the metal target more quickly than the spread of the debris plume, or the metal plate must move laterally past a fixed point of impact, carrying its plume with it. What is this speed, and how dense is this splash flow? Aside from its technological application, this problem has an intrinsic interest because it includes such a wide range of physical phenomena, and because it is an analog in miniature of supernova explosions. A small hot source created quickly propels an expanding flow into a vacuum, and this flow contains a remnant plasma that preserves information about the earliest moments of the flow.

The plan of this report is as follows. The section "Heat source and neutral flow" reviews the physics of an electron beam pulse that generates enthalpy, which in turn propels a flow of neutral target material. The section "Thermal plasma" shows the Saha equation used to estimate the initial degree of ionization in the fluid and the collisional ionization rate coefficient used. The section "Frozen flow" describes the criterion that indicates when plasma has ceased to be in thermodynamic equilibrium with the neutral flow. The section "An Example" shows a specific case that was originally measured and calculated at Los Alamos, and described in reference (2).

\section{Heat source and neutral flow}

An electron penetrating matter will deposit some of its energy into enthalpy directly by collisions, and by emitting bremsstrahlung radiation, a portion of which is absorbed. The details of this physics are drawn from Berger and Seltzer, (7) and (8), and Fermi (9), and are summarized in "Appendix 1: Energy loss by electrons." Thin metal plates of high atomic number $(\mathrm{Z})$ are used as radiation sources because they generate a higher proportion of radiation and absorb little of it. For thin plates, most of the heating is due to the collisional term of the energy deposition. The simplest estimate of this energy deposition was used in reference (1) and is

$$
(1 / \rho) d E / d x=1.6 \mathrm{MeV} \cdot \mathrm{cm}^{2} / \mathrm{gm}
$$


This value is within about $40 \%$ of the actual deposition in thin targets for electrons between 1 and $20 \mathrm{MeV}$ for a wide range of materials. A better estimate of energy deposition is given by using the formulas in Appendix 1, and by accounting for absorption of radiation by the target. Specific examples of collisional and radiative $(1 / \rho) d E / d x$ for aluminum and tantalum are shown in Appendix 1.

The report cited in reference (1) describes a model for determining the volume and temperature of hot fluid produced by electron beam "stopping power," $(1 / \rho) d E / d x$. The stagnation temperature (temperature at zero mass-average velocity) is the governing parameter, as the flow is assumed to be isentropic and a perfect gas. The flow within the plate is an unsteady, one-dimensional expansion, and on exiting, it continues as a two-dimensional supersonic expansion. This two-dimensional flow is treated in reference (1), and here, as a sequence of time intervals that each have a steady flow with a different mass flow rate. Flow at the exit areas, where the electron beam enters and exits the plate, is at Mach 1; the flow velocity equals the speed of sound.

Mach number is the parameter that ties all flow variables together. A refinement to the analysis in reference (1) is the use of the "stream-tube-area relations" to find the Mach number, temperature, velocity, and density of the two-dimensional expansion as functions of distance from the exit area. The stream-tube-area relations are based on the assumption that flow properties are constant across any cross section of a stream tube, and the stream tube outside the plate is assumed to bound a hemispherical expansion. The equation of mass conservation for a stream tube is

$$
\rho V A=\text { constant }=\rho_{*} C_{*} A_{*}
$$

for the product of density $\rho(R)$, velocity $V(R)$, and area $2 \pi R^{2}$, and where the star subscripts refer to conditions at the throat (one on each side of the plate), where $M=1$. See the work cited in reference (10) for a complete and elegant summary of compressible flow formulas. Shapiro (11) discusses the subject thoroughly. 
A formula for Mach number as a function of distance, $M(R)$, is derived by using the relationship between $\mathrm{A}_{*} / A$ and $M$ from reference (10), with $\mathrm{A}_{*} / A=\left(\pi \mathrm{r}_{0}^{2}\right) /\left(2 \pi R^{2}\right)$, and is

$$
M^{2}=\frac{1}{4}\left(\frac{\mathrm{r}_{0}}{R}\right)^{4}\left(\frac{2}{\gamma+1}\right)_{\gamma-1}^{\frac{\gamma+1}{\gamma+1}}\left(1+\frac{\gamma-1}{2} M^{2}\right)^{\frac{\gamma+1}{\gamma-1}}
$$

Here $\gamma$ is the ratio of specific heats ( $5 / 3$ for a monatomic gas), and $\pi r_{0}{ }^{2}$ is the area of the electron beam as it enters the plate. Only flow counter to the electron beam is considered. Flow is modeled for radial distances $R \geq \mathrm{r}_{0} / \sqrt{ } 2$, and Mach 1 flow is assumed to extend from $R=0$ to $R \leq \mathrm{r}_{0} / \sqrt{ } 2$. Figure 2 in reference (1) shows that this is physically reasonable. The temperature, velocity, and density of the flow at a given distance from the exit area are given by:

$$
\begin{aligned}
& \frac{T(R)}{\mathrm{T}_{0}}=\frac{1}{1+\frac{\gamma-1}{2} M(R)^{2}} \\
& \frac{V(R)}{\mathrm{c}_{0}}=\frac{M(R)}{\sqrt{1+\frac{\gamma-1}{2} M(R)^{2}}} \\
& \frac{\rho(R, t)}{\rho_{0}\left(t-\int_{0}^{R} \frac{d \xi}{V(\xi)}\right)}=\frac{1}{\left(1+\frac{\gamma-1}{2} M(R)^{2}\right)^{\frac{1}{\gamma-1}}}
\end{aligned}
$$

where $T_{0}$ is the stagnation temperature, $c_{0}$ is the stagnation speed of sound, and $\rho_{0}$ is the stagnation density at a delayed time that accounts for travel over distance $R$. The stagnation density drops as the source volume vents, see reference (1) for a detailed discussion.

At high Mach number, and for $\gamma=5 / 3$, the following relations with respect to $R$ occur: 


$$
\begin{gathered}
M(R)=4^{\frac{\gamma-1}{4}}\left(\frac{\gamma+1}{\gamma-1}\right)^{\frac{\gamma+1}{4}}\left(\frac{R}{\mathrm{r}_{0}}\right)^{\gamma-1}=3.1748\left(\frac{R}{\mathrm{r}_{0}}\right)^{\frac{2}{3}} \\
\frac{T(R)}{\mathrm{T}_{0}}=\frac{2}{\gamma-1} \frac{1}{M(R)^{2}}=0.2976\left(\frac{\mathrm{r}_{0}}{R}\right)^{\frac{4}{3}} \\
\frac{\mathrm{V}}{\mathrm{C}_{0}}=\sqrt{\frac{2}{\gamma-1}}=1.7321 \\
\frac{\rho(R, t)}{\rho_{0}\left(t-\frac{R}{\sqrt{\frac{2}{\gamma-1}} \mathrm{c}_{0}}=\left(\frac{2}{\gamma-1}\right)^{\frac{1}{\gamma-1}} \frac{1}{M(R)^{\frac{2}{\gamma-1}}}\right.}= \\
0.1624\left(\frac{\mathrm{r}_{0}}{R}\right)^{2}=\frac{\rho(R, t)}{\rho_{0}\left(t-\frac{R}{1.7321 \mathrm{c}_{0}}\right)}
\end{gathered}
$$

Already by $R=2 \mathrm{r}_{0}$ the flow has $\mathrm{M}>5$ and is at $94 \%$ of maximum velocity $1.7321 \mathrm{c}_{0}$. At $R=40 \mathrm{r}_{0}$ the flow has $\mathrm{M}=37$, the temperature ratio is 0.0022 , and the density ratio is 0.0001 . The analysis of the flow in reference (1) used the high Mach number approximation.

The difference between the neutral density $\rho(R, t)$ found with the stream-tube-area relations, equations (3), (4), (5), and (6), or the high Mach number approximations, equations (7), (8), (9), and (10), is negligible for examples typical of the radiography application. However, the quantity of plasma throughout the flow is largely determined by the steep gradients of flow properties near the exit area, and for this the accuracy of the stream-tube-area relations is essential.

\section{Thermal plasma}

The equation developed by Saha (1920) for the equilibrium concentration of plasma in a gas at temperature $T$ in ${ }^{\circ} \mathrm{K}$ is 


$$
\frac{\left(\frac{n_{+}}{N}\right)^{2}}{1-\left(\frac{n_{+}}{N}\right)^{2}} p=\frac{2 \mathrm{~g}_{+}}{\mathrm{g}_{\mathrm{N}}}\left(\frac{2 \pi \mathrm{m}_{\mathrm{e}}}{\mathrm{h}^{2}}\right)^{3 / 2}(\mathrm{k} T)^{5 / 2} \mathrm{e}^{-\mathrm{eV} \mathrm{i} / \mathrm{k} T}
$$

Equation (11) is in the form shown by Cobine (12) and also by Vincenti and Kruger (6). Here electron and ion densities are assumed equal $\left(n_{\mathrm{e}}=n_{+}\right)$, the gas pressure is $p=\mathrm{kNT}$ with number density $N=$ $\rho / \mathrm{m}_{\mathrm{N}}$ (for atomic mass $\mathrm{m}_{\mathrm{N}}$ ), the factor involving the ion degeneracy $\mathrm{g}_{+}$ and neutral degeneracy $g_{N}$ is assumed equal to one, $V_{i}$ is the ionization potential in $\mathrm{eV}, \mathrm{k}$ is Boltzmann's constant, $\mathrm{h}$ is Planck's constant, $\mathrm{m}_{\mathrm{e}}$ is the electron mass, and e is the elementary charge. For $T$ in units of $\mathrm{eV}$ the equilibrium concentration is

$$
\begin{aligned}
& \frac{\left(\frac{n_{+}}{N}\right)^{2}}{1-\left(\frac{n_{+}}{N}\right)^{2}}=\frac{1}{N} \frac{2 \mathrm{~g}_{+}}{\mathrm{g}_{\mathrm{N}}}\left(\frac{2 \pi \mathrm{m}_{\mathrm{e}} \mathrm{e}}{\mathrm{h}^{2}}\right)^{3 / 2} T^{3 / 2} \mathrm{e}^{-\mathrm{V}_{\mathrm{i}} / T} \equiv K(T, N) \\
& \frac{n_{+}}{N}=\sqrt{\frac{K(T, N)}{1+K(T, N)}} \equiv \phi(T, N)
\end{aligned}
$$

This restatement of Saha's equation is consistent with forms shown by Tanenbaum (13), and Mitchner and Kruger (14). For $T$ in $\mathrm{eV}$ and $N$ in $\mathrm{cm}^{-3}$, the function $K(T, N)$ is

$$
K(T, N)=\left(3.0183 \times 10^{21} \frac{1}{\mathrm{~cm}^{3} \mathrm{eV}^{3 / 2}}\right) \frac{1}{N} T^{3 / 2} \mathrm{e}^{-\mathrm{V}_{\mathrm{i}} / T}
$$

Electron collisional ionization is assumed to dominate photoionization and atomic collisional ionization because electrons maintain the highest collision frequency of any species during flow. A rate coefficient $\alpha$ for ionization by electron impact is

$$
\frac{\alpha}{N}=\sigma_{\mathrm{i}} \sqrt{\frac{8 \mathrm{e} T}{\pi \mathrm{m}_{\mathrm{e}}}}\left(1+\frac{\mathrm{V}_{\mathrm{i}}}{T}\right) \mathrm{e}^{-\mathrm{V}_{\mathrm{i}} / T}
$$


The rate coefficient $\alpha$ has the units of $\mathrm{s}^{-1}$. Units of $\mathrm{eV}$ are used for $T$ and $\mathrm{V}_{\mathrm{i}}$. Finally, $\sigma_{\mathrm{i}}=0.8798 \times 10^{-16} \mathrm{~cm}^{2}$, the cross section defined by the first Bohr orbit at radius $0.5292 \times 10^{-8} \mathrm{~cm}$. The cross section for ionization is assumed to be a step function with a threshold at $V_{i}$ and a magnitude of $\sigma_{i}$. This cross section model is a simplification suggested by the ionization cross section formulas by each of Drawin (1961) and Gryzinski (1965), which are described by Mitchner and Kruger (14). Equation (14) is the result of integrating the product of ionization cross section and electron thermal speed over a Maxwell electron energy distribution.

\section{Frozen flow}

Consider flow where $T$ and $N$ may depend on spatial coordinates but not on time; this is steady flow. From the perspective of a frame of reference moving with the mass-average velocity of the flow, the time rate of change of plasma density within a small parcel of fluid is

$$
\frac{\partial n_{\mathrm{e}}}{\partial t}=\alpha n_{\mathrm{e}}-P n_{\mathrm{e}}^{2}
$$

for ionization rate $\alpha n_{\mathrm{e}}$ and recombination rate $P n_{\mathrm{e}} n_{+}$. Only single ionization is assumed for simplicity. A parcel in local thermodynamic equilibrium will have a concentration of plasma $n_{\mathrm{e}}=n_{+}=\alpha / P$, where the rate coefficients $\alpha$ and $P$ each depend on $T$ and $N$. If the plasma density is perturbed from equilibrium by a small quantity $\eta$, then

$$
\begin{aligned}
& n_{\mathrm{e}}=\frac{\alpha}{P}+\eta \\
& \frac{\partial n_{\mathrm{e}}}{\partial t}=\alpha n_{\mathrm{e}}-P n_{\mathrm{e}}^{2} \rightarrow \frac{\partial \eta}{\partial t}=-\alpha \eta-P \eta^{2} \\
& \eta(t) \approx \eta(0) \mathrm{e}^{-\alpha t}
\end{aligned}
$$

where small terms of second order are neglected. The ionization rate coefficient is the measure of the responsiveness of the fluid to perturbations from equilibrium of its plasma density. 
The equation of continuity for the plasma species from the perspective of a static frame of reference is

$$
\frac{\partial n_{\mathrm{e}}}{\partial t}+\nabla \cdot n_{\mathrm{e}} \mathbf{V}=0
$$

where $\mathrm{V}$ is the mass-average velocity and plasma diffusion is neglected, and there are no external sources or sinks of plasma. In steady equilibrium flow the local rate-dependent processes compensate for any local inflow or outflow of plasma density, so equation (17) can be restated as

$$
\alpha n_{\mathrm{e}}-P n_{\mathrm{e}}^{2}+\nabla \cdot n_{\mathrm{e}} \mathrm{V}=0
$$

Rate-dependent processes are so fast in equilibrium flow that they easily adjust to any changes introduced by transport. If $\mathrm{L}_{\mathrm{n}}$ is a length scale for the variation of density, and $V$ is a characteristic flow velocity, then $\alpha \mathrm{L}_{\mathrm{n}} / V>>1$. This nondimensional parameter is the exponential in equation (16) when $t=\mathrm{L}_{\mathrm{n}} / V$, the time scale for the variation of density.

The temperature and density drop so precipitously during the rapid expansion into the vacuum, that both the ionization and recombination rate coefficients decrease by orders of magnitude over a short distance. The steady flow far downstream from the source is a simple geometric expansion at constant velocity,

$$
\sqrt{\frac{2}{\gamma-1}} \frac{\mathrm{c}_{0}}{R^{2}} \frac{d}{d R}\left(R^{2} n_{\mathrm{e}}\right)=0
$$

and with $\alpha \mathrm{L}_{n} / V \ll 1$, the plasma density is "frozen." The frozen plasma density is well above what would be expected from local thermodynamic conditions because the three-body recombination reaction no longer occurs in the cold and rarefied flow.

The transition from equilibrium flow to frozen flow is sudden, and it can be approximated as occurring at a single hemispherical cross section of the expansion. At this transition point, recombination 
is negligible and the rate of change due to ionization is comparable to the rate of change due to transport, or equivalently $\alpha \mathrm{L}_{n} / V=1$. The criterion for determining the point of transition is

$$
\alpha n_{\mathrm{e}}+\nabla \cdot n_{\mathrm{e}} \mathrm{V}=0=\alpha n_{\mathrm{e}}+\frac{1}{R^{2}} \frac{d}{d R}\left(R^{2} n_{\mathrm{e}} V\right)
$$

an idea originally proposed by Bray, see references (4), (5), and (6). Note that the quantities in equation (20) are calculated on the basis of equilibrium flow. Conditions prior to the transition are taken to be in complete equilibrium, and conditions after the transition are assumed frozen.

An explicit formula for the coordinate $\mathrm{R}_{\mathrm{B}}$ at the point of transition is derived from equation (20) by using the stream-tubearea relations to specify $V[M(R)], T[M(R)], N[M(R), t]$, the Saha equation for $n_{\mathrm{e}}$, and a formula for the ionization coefficient $\alpha(T, N)$. This result is

$$
\mathrm{R}_{\mathrm{B}}=\frac{2 \mathrm{~V}}{\alpha}\left\{\left[(\gamma-1)\left(1-\phi^{2}\right)\left(\frac{3}{2}+\frac{\mathrm{V}_{\mathrm{i}}}{\mathrm{T}}\right)-1-\phi^{2}-\frac{2}{\mathrm{M}^{2}}\right] \frac{\mathrm{M}^{2}}{(3 \gamma+5) \mathrm{M}^{2}-1}-1\right\}
$$

where all quantities are calculated on the basis of equilibrium flow at coordinate $R=\mathrm{R}_{\mathrm{B}}$. These quantities are the velocity $\mathrm{V}$, ionization rate coefficient $\alpha$, ionization fraction $\phi$, temperature $\mathrm{T}$ in $\mathrm{eV}$, and Mach number M. A summary of the derivation is presented in "Appendix 2: Bray criterion." The implications of equation (21) are easier to visualize by considering the limit of high Mach number $\left(\mathrm{M}^{2}>2\right)$, low ionization fraction $\left(\phi^{2}<1\right)$, and temperature well below the ionization potential $\mathrm{V}_{\mathrm{i}}$,

$$
\mathrm{R}_{\mathrm{B}} \approx \frac{2 \mathrm{~V}}{\alpha}\left\{\frac{\gamma-1}{3 \gamma+5} \frac{\mathrm{V}_{\mathrm{i}}}{\mathrm{T}}-1\right\}=\frac{2 \mathrm{~V}}{\alpha}\left\{\frac{1}{15} \frac{\mathrm{V}_{\mathrm{i}}}{\mathrm{T}}-1\right\}
$$

The distance between the exit area $(M=1)$ and the transition to frozen flow diminishes as the freezing temperature increases, up to the limit $(\gamma-1) \mathrm{V}_{\mathrm{i}} /(3 \gamma+5)$. The freezing temperature for this $\gamma=5 / 3$ 
flow has an upper limit of $\mathrm{V}_{\mathrm{i}} / 15$. For aluminum this limit is $0.4 \mathrm{eV}$, and for tantalum it is $0.5 \mathrm{eV}$. If the freezing temperature is at half of the maximum value then $R_{B} \approx 2 V / \alpha$, or equivalently $\alpha R_{B} / V \approx 2$.

\section{An Example}

A specific example of these phenomena is the experiment with an aluminum target described by DeVolder and her colleagues (2). An electron beam of $3.8 \mathrm{kA}$ at $5.25 \mathrm{MeV}$ within a diameter of $1 \mathrm{~mm}$ and a pulse width of $70 \mathrm{~ns}$ strikes an aluminum plate $0.8 \mathrm{~mm}$ thick. Figures $1,2,3$, and 4 show the $\log$ of the neutral density, the log of the plasma density, the log of the ionization fraction, and the electron temperature for this example as calculated by using the formulas in this report. I will use this example to illustrate the model presented here.

As shown in Appendix 1, electrons lose $(1 / \rho) d E / d x=1.657$ $\mathrm{MeV} \cdot \mathrm{cm}^{2} / \mathrm{gm}$, of which 1.527 (92\%) is a collision loss and $0.130(8 \%)$ is a radiative loss. Assuming bremsstrahlung photons have an average mean-free-path $\lambda_{v}=1.4 \mathrm{~cm}$, then $1.3 \%$ of the radiative loss contributes to material enthalpy. This estimate of absorption is described in "Appendix 3: Radiation capture." The stagnation temperature is found to be $\mathrm{T}_{0}=2.85 \mathrm{eV}$ by using $(1 / \rho) d E / d x=1.529 \mathrm{MeV} \cdot \mathrm{cm}^{2} / \mathrm{gm}$ in the formula shown in reference (1).

Figure 5 shows the log of the neutral density as a function of time at the exit area. This is the result of the one-dimensional, clumped-wave analysis of the flow within the metal plate. The report cited in reference (1) is largely devoted to describing this clumpedwave analysis. The flow at the exit areas is at Mach 1, the ratio of the density there to the stagnation density is 0.6495 . This is the source history seen by the external two-dimensional flow. It is very convenient to model this two-dimensional flow as a sequence of steady flows each at a mass flow rate set by the "steps" of Figure 5. The mass front, seen as the cliff in Figure 1, moves at a speed of $V=$ $7.11 \mathrm{~mm} / \mu \mathrm{s}$, except very close to the $\mathrm{M}=1$ area, where $V=3.56$ $\mathrm{mm} / \mu \mathrm{s}$. 
The ionization fraction and the quantities included in the Bray criterion shown as equation (21) are now calculated throughout the equilibrium flowfield. From this information the self-consistent trajectory $R=\mathrm{R}_{\mathrm{B}}(R, t)$, the location of the transition from equilibrium to frozen flow over the course of time, is determined. Flow that has yet to reach this space-time boundary line has plasma in Saha equilibrium with the neutral flowfield. As parcels of fluid cross the space-time boundary line their ionization fractions are fixed to the ionization fractions they experienced at the transition point. This is also true for the electron temperature. Figure 6 shows the ionization fraction and the location of the transition point over the course of time for this example. Frozen supersonic flow is described by hyperbolic partial differential equations, and the trajectory of the Bray criterion is their space-time boundary condition. The ionization fraction in the frozen part of the flowfield is specified by a method-of-characteristics propagation of the transition boundary line information. This "raylike" nature of the frozen flow is clearly visible in Figures 2, 3 and 4. Figure 4 shows frozen electron temperatures of $0.24 \mathrm{eV}$ and $0.31 \mathrm{eV}$, a little over half of the maximum of $0.4 \mathrm{eV}$ found from equation (22).

Figures 7 and 8 show radial profiles of the neutral and plasma densities at times $1.52 \mu$ s and $2.9 \mu$ s, respectively. At $1.52 \mu$ s the matter front is essentially a shell about $3 \mathrm{~mm}$ thick of atmospheric density metal $\left(1 \mathrm{ATM}=2.69 \times 10^{19}\right.$ atoms $\left./ \mathrm{cm}^{-3}\right)$. The ionization fraction is about $10^{-4}$. At $2.9 \mu \mathrm{s}$ the flow extends to $R=2 \mathrm{~cm}$, the leading edge is at $22 \%$ of atmospheric density, and plasma density is between $10^{11} \mathrm{~cm}^{-3}$ and $10^{14} \mathrm{~cm}^{-3}$ along the profile. That plasma is out of thermal equilibrium is clear when you consider that the temperature of the neutral flow at $R=1 \mathrm{~cm}$ is $T=178{ }^{\circ} \mathrm{K}$, and at $R=2$ $\mathrm{cm}$ the neutral temperature is $T=72^{\circ} \mathrm{K}$.

The Los Alamos results for this example are as follows:

1) The measured velocity is $V=8.1 \mathrm{~mm} / \mu \mathrm{s}$,

2) the stagnation temperature peaks at $1.9 \mathrm{eV}$,

3 ) the electron temperature is between $0.2 \mathrm{eV}$ and $0.34 \mathrm{eV}$ (at $2 \mu \mathrm{s}$ ),

4) the leading edge density for $R \approx 1 \mathrm{~cm}$ and $1.25 \mu \mathrm{s} \leq t \leq 1.50 \mu \mathrm{s}$ is $\rho / \rho_{0} \approx 5 \times 10^{-4}$ of solid density (so $N \approx 3 \times 10^{19}$ atoms $/ \mathrm{cm}^{-3}$ as solid aluminum $=2.699 \mathrm{gm} / \mathrm{cm}^{-3}=6.024 \times 10^{22}$ atoms $\left./ \mathrm{cm}^{-3}\right)$, 
5 ) and the density of electrons is $n_{\mathrm{e}} \approx 1 \times 10^{17} \mathrm{~cm}^{-3}$ for $R \approx 0.4 \mathrm{~cm}$ and $t=0.5 \mu \mathrm{s}$.

Velocity is the only measured quantity of the Los Alamos results just described. DeVolder's report (2) did not show plasma density beyond $0.5 \mu \mathrm{s}$. The analytical model of this report gives a plasma density of $n_{\mathrm{e}}$ $\approx 3 \times 10^{16} \mathrm{~cm}^{-3}$ for the front at $R \approx 0.3 \mathrm{~cm}$ and $t \approx 0.5 \mu \mathrm{s}$. All these parameters compare quite well.

The clearest difference between the Los Alamos calculations and the model here is in the neutral density profiles. While the leading edges of the Los Alamos profiles are similar to those shown here, the Los Alamos calculations show a density maximum at $R=1 \mathrm{~cm}$ and $t \approx$ $2.0 \mu \mathrm{s}$. Even before this time, the density between the plate and the front in the Los Alamos work is much higher than in the model here. I have no explanation; however, the Los Alamos calculations include more physical effects than I considered. In particular, the source volume will exert pressure on the cold plate, enlarging itself at the expense of stagnation temperature (by " $p d V$ ” work). This enlargement works against the resistance to deformation, fracture, and ablation of the solid plate. It is possible that within a microsecond these effects have introduced denser material into the flowfield. As in all good physics problems, there is always more to learn.

\section{Appendix 1: Energy loss by electrons}

The energy lost per unit length through collisions by an electron while penetrating matter is given by Bethe's stopping-power theory, shown below in the formulation quoted by Berger and Seltzer (7):

$$
\begin{aligned}
& -\frac{1}{\rho}\left(\frac{d E}{d x}\right)=\frac{2 \pi \mathrm{Na}_{\mathrm{a}} \mathrm{r}_{\mathrm{e}} \mathrm{mc}^{2}}{\beta} \frac{\mathrm{Z}}{\mathrm{A}}\left\{\ln \left[\frac{\tau^{2}(\tau+2)}{2\left(\mathrm{I} / \mathrm{mc}^{2}\right)^{2}}\right]+F(\tau)-\delta\right\} \\
& F(\tau)=1-\beta^{2}+\left[\tau^{2} / 8-(2 \tau+1) \ln (2)\right] /(\tau+1)^{2} \\
& \delta=\log \left(\frac{\beta^{2}}{1-\beta^{2}}\right)
\end{aligned}
$$




$$
\begin{aligned}
& I=\left(9.76+\frac{58.8}{Z^{1.19}}\right) \mathrm{Z} \mathrm{eV} \\
& \beta=\sqrt{1-\frac{1}{(\tau+1)^{2}}}
\end{aligned}
$$

The various symbols have the following meanings: $\tau$ is the electron kinetic energy in units of $\mathrm{mc}^{2}(0.510976 \mathrm{MeV}), \beta$ is the ratio of the electron velocity to the speed of light (c), I is the mean excitation (ionization) energy in $\mathrm{eV}$ [see references (7), (8), and (9)], Z is the atomic number, $A$ is the atomic weight, $\mathrm{r}^{2}=\left(\mathrm{e}^{2} / \mathrm{mc}^{2}\right)^{2}=7.9403$ $\times 10^{-26} \mathrm{~cm}^{2}, \mathrm{~N}_{\mathrm{a}}=6.02486 \times 10^{23}$ electron/mole (Avogadro's number in the old mass scale in which the atomic weight of $\mathrm{O}^{16}$ is exactly 16), $\delta$ is an approximation to the density effect correction shown in reference (7), and $\rho$ is the mass density in $\mathrm{gm} / \mathrm{cm}^{3}$.

Electrons also lose energy by radiation as they traverse matter. The average radiative loss is:

$$
-\frac{1}{\rho}\left(\frac{d E}{d x}\right)_{\mathrm{rad}}=\frac{4 \mathrm{~N}_{\mathrm{a}} \mathrm{r}_{\mathrm{e}}^{2} \mathrm{Z}^{2} E}{137 \mathrm{~A}} \ln \left(183 \mathrm{Z}^{-1 / 3}\right)
$$

Fermi (9) shows the ratio of radiative energy loss to ionization loss as given roughly by $y_{F}$,

$$
y_{\mathrm{F}}=[\mathrm{Z} \times(\mathrm{MeV})] / 800
$$

Energy loss by radiation equals the energy loss by collision $\left(y_{\mathrm{F}}=1\right)$ at the critical energy, shown by Berger and Seltzer (7) as

$$
\mathrm{E}_{\text {crit }}=800 /(\mathrm{Z}+1.2) \mathrm{MeV}
$$

The total energy loss is $\left(1+y_{\mathrm{F}}\right)$ times the collision loss shown as (A1.1), the ratio of collision loss to total loss is $1 /\left(1+y_{\mathrm{F}}\right)$, and the ratio of radiative loss to total loss is $y_{\mathrm{F}} /\left(1+y_{\mathrm{F}}\right)$. 
As an example, a $5.25 \mathrm{MeV}$ electron penetrating aluminum loses $(1 / \rho) d E / d x=1.657 \mathrm{MeV} \cdot \mathrm{cm}^{2} / \mathrm{gm}$, of which $1.527(92 \%)$ is a collision loss and $0.130(8 \%)$ is a radiative loss. At $20 \mathrm{MeV}$ the total loss is 2.29 $\mathrm{MeV} \cdot \mathrm{cm}^{2} / \mathrm{gm}$, of which $1.728(75.5 \%)$ is a collision loss and 0.562 $(24.5 \%)$ is a radiative loss. For aluminum the critical energy is 56.3 $\mathrm{MeV}$.

For tantalum at $5.25 \mathrm{Mev}$, the total loss is $1.612 \mathrm{MeV} \cdot \mathrm{cm}^{2} / \mathrm{gm}$, of which $1.090(67.6 \%)$ is a collision loss and $0.522(32.4 \%)$ is a radiative loss. At $20 \mathrm{MeV}$ the total loss is $3.558 \mathrm{MeV} \cdot \mathrm{cm}^{2} / \mathrm{gm}$, of which $1.259(35.4 \%)$ is a collision loss and $2.299(64.6 \%)$ is a radiative loss. For tantalum the critical energy is $10.8 \mathrm{MeV}$.

The energy deposited by an electron that raises the enthalpy of the material is the collision loss plus the absorbed fraction of the radiative loss. The estimate used in reference (1) for the electron energy deposited into enthalpy was $1.6 \mathrm{MeV} \cdot \mathrm{cm}^{2} / \mathrm{gm}$.

\section{Appendix 2: Bray criterion}

The transition from equilibrium to frozen flow occurs when

$$
\frac{d}{d R}\left(V n_{\mathrm{e}} R^{2}\right)+\alpha n_{\mathrm{e}} R^{2}=\frac{d}{d R}\left(V R^{2} N \phi\right)+\alpha R^{2} N \phi=0
$$

At the point of transition, $R=\mathrm{R}_{\mathrm{B}}$,

$$
\frac{-2}{R_{B}}=\frac{\alpha}{V}+\frac{1}{\phi} \frac{d \phi}{d R}+\frac{1}{V} \frac{d V}{d R}+\frac{1}{N} \frac{d N}{d R}
$$

where all quantities are based on equilibrium flow at coordinate $\mathrm{R}_{\mathrm{B}}$. The derivatives in $R$ are converted to derivatives in Mach number,

$$
\frac{-2}{R_{B}}=\frac{\alpha}{V}+\left\{\frac{1}{\phi}\left[\frac{\partial \phi}{\partial T} \frac{d T}{d M}+\frac{\partial \phi}{\partial N} \frac{d N}{d M}\right]+\frac{1}{V} \frac{d V}{d M}+\frac{1}{N} \frac{d N}{d M}\right\} \frac{d M}{d R}
$$

The derivatives in (A2.3) are evaluated by using stream-tube-area relations for each of M, T, V, and N, and the Saha equation for $\phi$. These 
equations are numbered in the text as follows: (3), (4), (5), (6), and (12). The result is

$\mathrm{R}_{\mathrm{B}}=\frac{2 \mathrm{~V}}{\alpha}\left\{\left[(\gamma-1)\left(1-\phi^{2}\right)\left(\frac{3}{2}+\frac{\left.\mathrm{V}_{\mathrm{i}}\right)}{\mathrm{T}}-1-\phi^{2}-\frac{2}{\mathrm{M}^{2}}\right] \frac{\mathrm{M}^{2}}{(3 \gamma+5) \mathrm{M}^{2}-1}-1\right\}\right.$

and is shown as equation (21) in the text. In any particular case the numerical value of $R_{B}$ is the root of (A2.4).

In deriving equation (A2.4), the stagnation number density $\mathrm{N}_{0}$, which appears in equation (6), is treated as a constant. See reference (1) for a description of this "clumped wave" model of the source flow. Shapiro (11) uses the phrase "lumped pressure waves." In the clumped wave model, the stagnation density is constant during the time an expansion wave travels at the speed of sound from one end of the source region in the metal plate to the other. When an expansion wave reflects at the exit area back into the source then the mass flow rate out (at $M=1$ ) readjusts "instantly" to a lower value that remains constant until the next traversal is complete. The stagnation density history seen by the external flow is a downward staircase in time, the source density does not change with time except at the instants of wave reflection at the exit areas.

The ionization fraction in any parcel of fluid becomes a constant as the parcel crosses the transition. The history of the ionization fraction at the locations of transition, $\phi\left[R_{\mathrm{B}}(t)\right]$, forms the boundary condition for the method-of-characteristics solution of the subsequent frozen flow. See references (3), (4), (5), and (6) for discussions of the transition from equilibrium to "frozen" nonequilibrium flow, and the Bray criterion.

\section{Appendix 3: Radiation capture}

A portion of the bremsstrahlung emission generated by the electron beam is captured by the target plate and converted into heat. Thin, high $\mathrm{Z}$ plates are used as radiation sources because they convert a higher proportion of the electron energy into radiation and they 
absorb little of it. What follows is a simple estimate of the fraction of the radiation captured by the plate.

Given a plate of thickness $\Delta \mathrm{L}$, an electron beam diameter of $\mathrm{d}_{0}$, and an average photon mean-free-path in the target of $\lambda_{v}$, then an estimate for the fraction of radiation captured is

$$
\frac{\frac{\Delta \mathrm{L}}{\mathrm{d}_{0}}}{1+\frac{\Delta \mathrm{L}}{\mathrm{d}_{0}}}\left(1-\mathrm{e}^{-\frac{\Delta \mathrm{L}}{2 \lambda_{v}}}\right)
$$

The first factor in (A3.1) is the fraction of the radiation that interacts with the plate, and the second factor is the fraction of interacting radiation that is absorbed. If the source region is a disc, then none of the radiation interacts, and if the source region is a line, then all of the radiation interacts. Photons that interact are assumed to travel an average distance $\Delta \mathrm{L} / 2$ through the plate.

Most of the bremsstrahlung radiation from multi-MeV electrons is in the x-ray portion of the spectrum. In the range of $50 \mathrm{keV}$ to 200 $\mathrm{keV}$, the photon mean-free-path in aluminum is between $1 \mathrm{~cm}$ and 3 $\mathrm{cm}$, and in tantalum it is between $0.01 \mathrm{~cm}$ and $0.08 \mathrm{~cm}$. Plechaty, Cullen, and Howerton (15) have tabulated photon mean-free-path data. 


\section{References}

(1) M. Garcia, "Splash flow from a metal plate hit by an electron beam pulse," UCRL-ID-128660, 4 September 1997.

(2) B. T. DeVolder, T. J. T. Kwan, R. D. Fulton, D. C. Moir, D. M. Oro, D. S. Prono, "Computational and experimental studies of the beamtarget interaction for high-dose, multi-pulse radiography," Los Alamos National Laboratory, LA-UR-97-8, and the 24th IEEE International Conference on Plasma Science, 7P26, May 19-22, 1997, San Diego, CA.

(3) Y. B. Zel'dovich and Y. P. Raizer, Physics of Shock Waves and High-Temperature Hydrodynamic Phenomena, in two volumes, W. D. Hayes and R. F. Probstein, editors, New York: Academic Press, 1996 and 1967. See:

1) Volume 1, chapter 1 , section 28, "Sudden isentropic expansion of a spherical gas cloud into vacuum," section 29, "Conditions for self-similar sudden expansion of a gas cloud into vacuum."

2) Volume 2, chapter 8, topic 3 "Disturbance of thermodynamic equilibrium in the sudden expansion of a gas into vacuum," section 6, "Sudden expansion of a gas cloud," section 7, "Freezing effect," section 8, "Disturbance of ionization equilibrium," section 9, "The kinetics of recombination and cooling of the gas following the disturbance of ionization equilibrium."

(4) K. N. C. Bray, "Atomic Recombination in a Hypersonic WindTunnel Nozzle," Journal of Fluid Mechanics, vol. 6, pt. 1, p. 1, 1959.

(5) K. N. C. Bray, "Electron-Ion Recombination in Argon Flowing through a Supersonic Nozzle," in The High Temperature Aspects of Hypersonic Flow, W. C. Nelson, editor, New York: Macmillan, 1964. 
(6) W. G. Vincenti and C. H. Kruger, Jr., Introduction to Physical Gas Dynamics, New York: John Wiley and Sons, Inc., 1965. See Chapter 8, section 13, "Nozzle Flow."

(7) M. J. Berger and S. M. Seltzer, Studies in penetration of charged particles in matter, National Bureau of Standards Nuclear Science Series Report No. 39, Washington DC: National Academy of Sciences-National Research Council Publication 1133, 1964.

(8) M. J. Berger and S. M. Seltzer, Stopping Powers and Ranges of Electrons and Positrons (2nd Ed.), NBSIR 82-2550-A, US Department of Commerce, National Bureau of Standards, February 1983.

(9) E. Fermi, Nuclear Physics, Revised Ed., University of Chicago Press, 1974.

(10) Equations, Tables, and Charts for Compressible Flow, National Advisory Committee for Aeronautics (NACA), Report 1135, Ames Aeronautical Laboratory, Moffett Field, CA, 1953.

(11) A. H. Shapiro, The Dynamics and Thermodynamics of Compressible Flow, Volume 1, New York: Ronald Press Company, 1953.

(12) J. D. Cobine, Gaseous Conductors, New York: Dover Publications, Inc., 1958.

(13) B. S. Tanenbaum, Plasma Physics, New York: McGraw-Hill Book Co., 1967.

(14) M. Mitchner and C. H. Kruger, Jr., Partially Ionized Gases, New York: John Wiley \& Sons, Inc., 1973.

(15) E. F. Plechaty, D. E. Cullen, and R. J. Howerton, "Tables and Graphs of Photon-Interaction Cross Sections from $0.1 \mathrm{keV}$ to $100 \mathrm{MeV}$ Derived from LLL Evaluated-Nuclear-Data Library," UCRL50400, Vol. 6, Rev. 3, November 11, 1981. 


\section{Figure legends}

(1) Log of neutral density in $\mathrm{cm}^{-3}$. The neutral density of aluminum for the example described over a domain extending to $2 \mathrm{~cm}$ and $2.9 \mu \mathrm{s}$. The large "cliff" is the material front advancing from left rear to right front at $7.11 \mathrm{~mm} / \mu \mathrm{s}$, after a rapid acceleration from $3.56 \mathrm{~mm} / \mu \mathrm{s}$ at the sonic origin. The sharp steps on this density surface are an artifact of the clumped-wave analysis of the flow within the metal plate, see reference (1). Density before the cliff is set arbitrarily to avoid plotting $\log (0)$.

(2) Log of electron density in $\mathrm{cm}^{-3}$. This is the density of plasma for the flow of Figure 1. Near the plate, plasma is in thermal equilibrium and it diminishes quickly as it flows. On crossing a transition point where recombination ceases, the ionization fraction is "frozen" into the flow and propagates downstream in the manner of a Riemann invariant of a field governed by hyperbolic partial differential equations. The "shelf" of nonequilibrium plasma is clearly visible in this figure, as are the "rays" of flow aligned with the material front. Density before the cliff is set arbitrarily to avoid plotting $\log (0)$.

(3) Log of ionization fraction. This figure shows the ionization fraction over the flowfield of Figures 1 and 2. The steep drop in the equilibrium region near the plate is in sharp contrast to the frozen region, which is "terraced" along flow characteristics. The ionization fraction steadily increases over time because the neutral density is diminishing while the local temperature is fixed. Ionization fraction before the cliff is set arbitrarily to avoid plotting $\log (0)$. The negative signs for the numbers on the vertical axis are fat due to computer gremlins.

(4) Electron temperature in $\mathrm{eV}$. This figure shows the electron temperature over the flowfield of the previous figures. The temperature at Mach 1 is $2.116 \mathrm{eV}$, and it drops precipitously through the equilibrium flow. The two plateaus of frozen electron temperature are a narrow one at $0.24 \mathrm{eV}$ and a broad one at $0.31 \mathrm{eV}$. The edges of these plateaus are aligned with flow characteristics. 
(5) Log neutral density ratio at $M=1$. This is the history of the outflow density at the sonic origin, a result of the clumped-wave analysis described in reference (1). Point locations for this calculation are shown. The clumped-wave analysis makes it possible to model the subsequent two-dimensional, supersonic flow as a sequence of steady flows, each of which has its mass flow rate set by the constant density appropriate to the time interval during which each flow issued from the plate.

(6) Ionization fraction at transition point, and the radial location of the transition point. These time charts show the ionization fraction at the location of the "Bray transition" during the course of the flow shown in the previous figures. The trajectory of this transition point is the space-time boundary condition to the hyperbolic partial differential equations describing the frozen flow. This boundary line, $R_{\mathrm{B}}(t)$, is the result of iteration because it is defined in terms of equilibrium flow properties at its own location. From the perspective of a particular parcel of fluid, the transition point is found by integrating the equations of motion and ionization-recombination rate equations forward in time until the Bray criterion is met. The approximation of a point for what in reality is a zone of transition is successful because the change is so sudden.

(7) Radial profiles of neutral and plasma densities. This is a radial cross section of Figures 1 and 2 at time $t=1.52 \mu \mathrm{s}$. The neutral density is seen to be essentially a shell of about $3 \mathrm{~mm}$ thickness and atmospheric density. The plasma density is more uniform at between $10^{14} \mathrm{~cm}^{-3}$ and $10^{15} \mathrm{~cm}^{-3}$.

(8) Radial profiles of neutral and plasma densities. This is a radial cross section of Figures 1 and 2 at time $t=2.91 \mu \mathrm{s}$. The neutral density is seen to be greatest at the leading edge, at about $15 \%$ of atmospheric density in the forward $3 \mathrm{~mm}$. The plasma density lies between $10^{14} \mathrm{~cm}^{-3}$ near the leading edge to $10^{12}$ $\mathrm{cm}^{-3}$ close to the plate. The gas flow for this example evolves into an expanding shell because the source has been exhausted. 


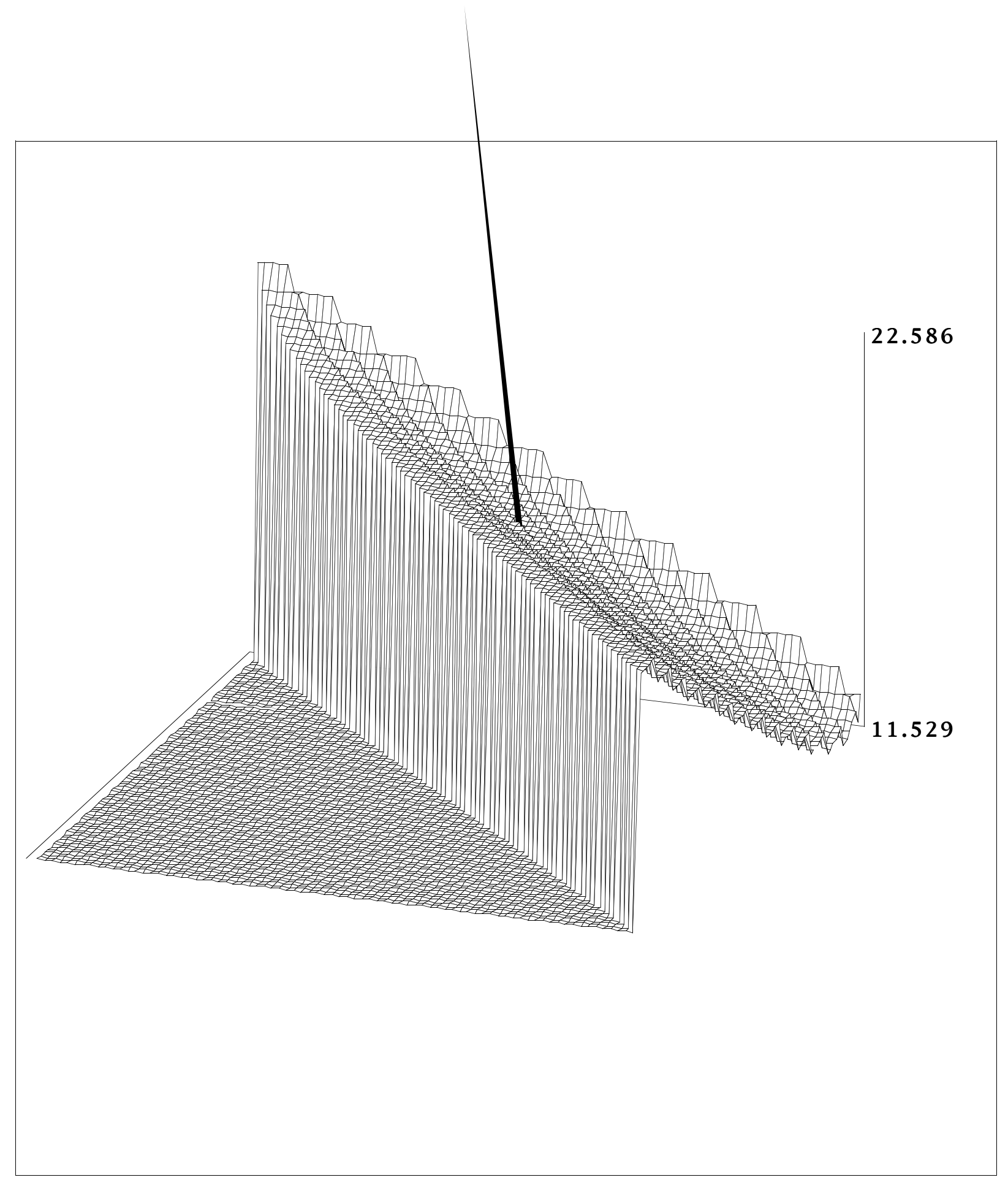

Lp

Figure 1: $\log$ of neutral density in $\mathrm{cm}^{\wedge}-3$

Radial axis is on the left, time axis is in back, domain extends from $(0,0)$ (at left rear)

to $(2 \mathrm{~cm}, 2.9 \mu \mathrm{s})$ (at right front) 




$\operatorname{Ln}$ e

Figure 2: $\log$ of electron density in $\mathrm{cm}^{\wedge}-3$

Radial axis is on the left, time axis is in back, domain extends from $(0,0)$ to $(2 \mathrm{~cm}, 2.9 \mu \mathrm{s})$ 




$\mathrm{L} \psi$

Figure 3: $\log$ of ionization fraction

Radial axis is on the left, time axis is in back, domain extends from $(0,0)$ to $(2 \mathrm{~cm}, 2.9 \mu \mathrm{s})$ 


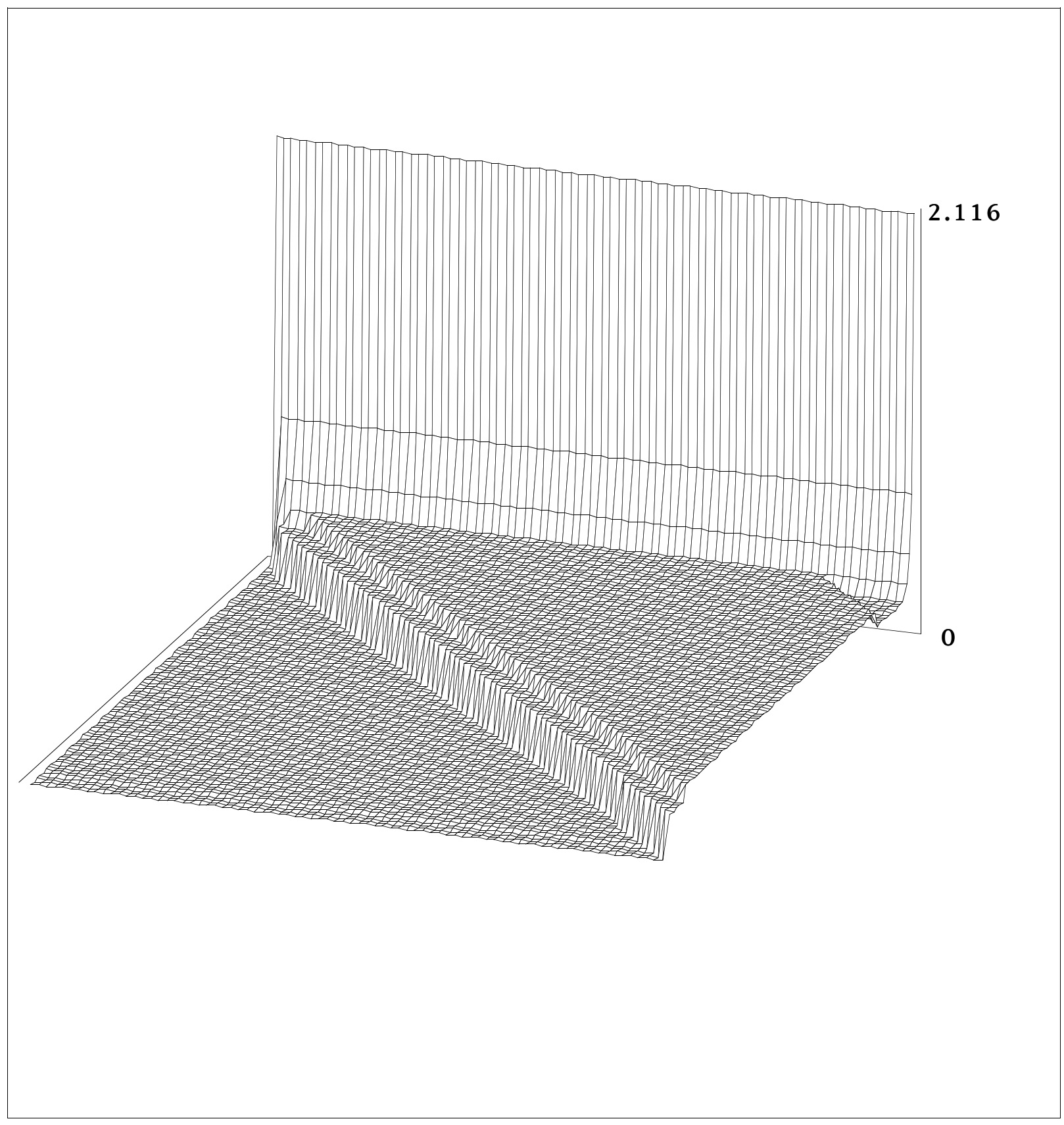

$\mathrm{T}_{\mathbf{f}}$

Figure 4: electron temperature in $\mathrm{eV}$

Radial axis is on the left, time axis is in back, domain extends from $(0,0)$ to $(2 \mathrm{~cm}, 2.9 \mu \mathrm{s})$, temperature plateaus at $0.24 \mathrm{eV}$ and $0.31 \mathrm{eV}$. 


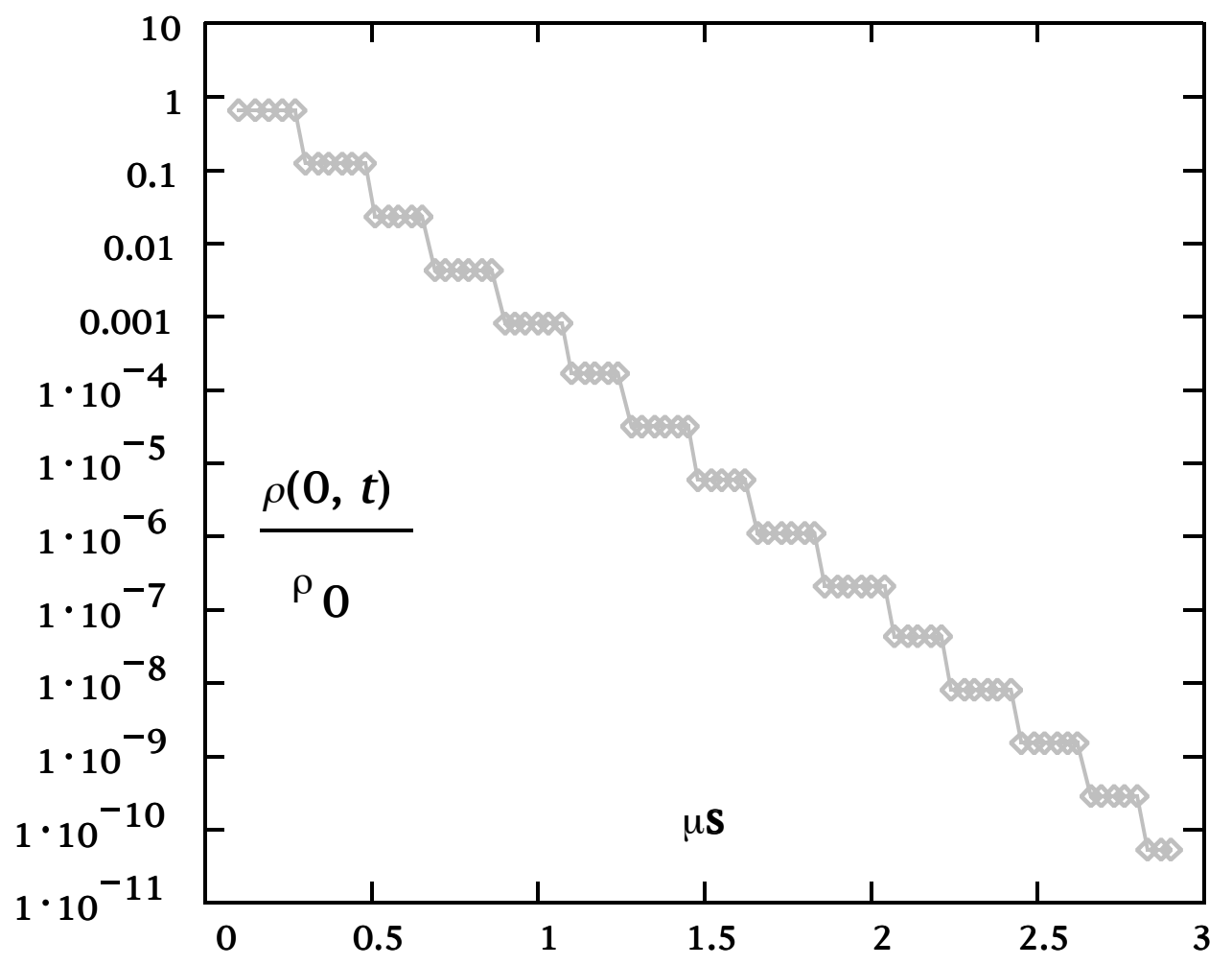

Figure 5: $\log$ neutral density ratio at $M=1$

Density at Mach 1 normalized by original density, time in $\mu$ s, point locations shown 

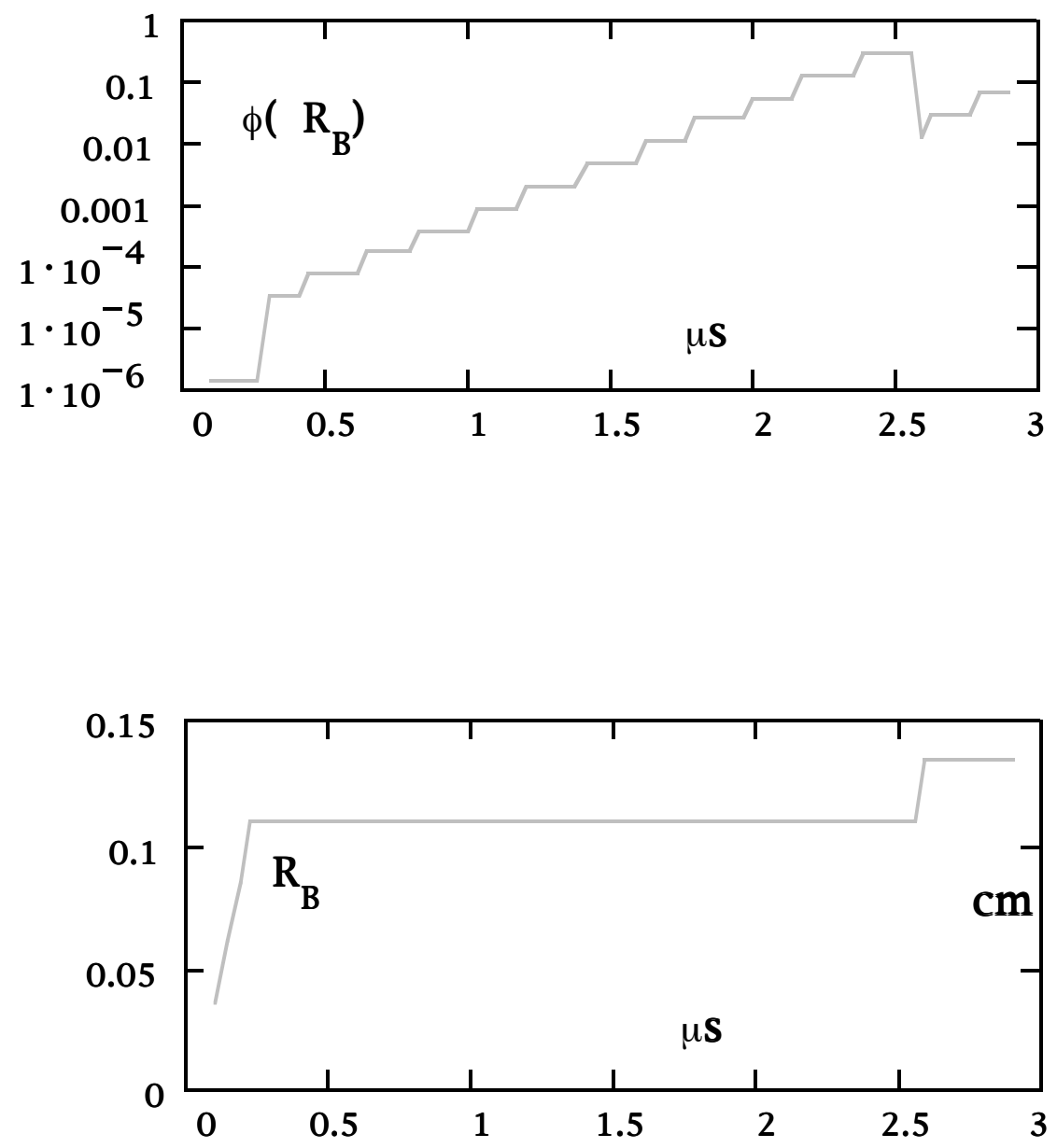

Figure 6: ionization fraction at transition point, and the radial location of the transition point The Bray criterion as a function of time:

the location where a specific ionization fraction is frozen for flow downstream from that point 


$$
\text { Time }=\operatorname{tr}_{\frac{\mathrm{NT}}{2}}=1.519 \cdot 10^{-6} \quad \text { seconds }
$$

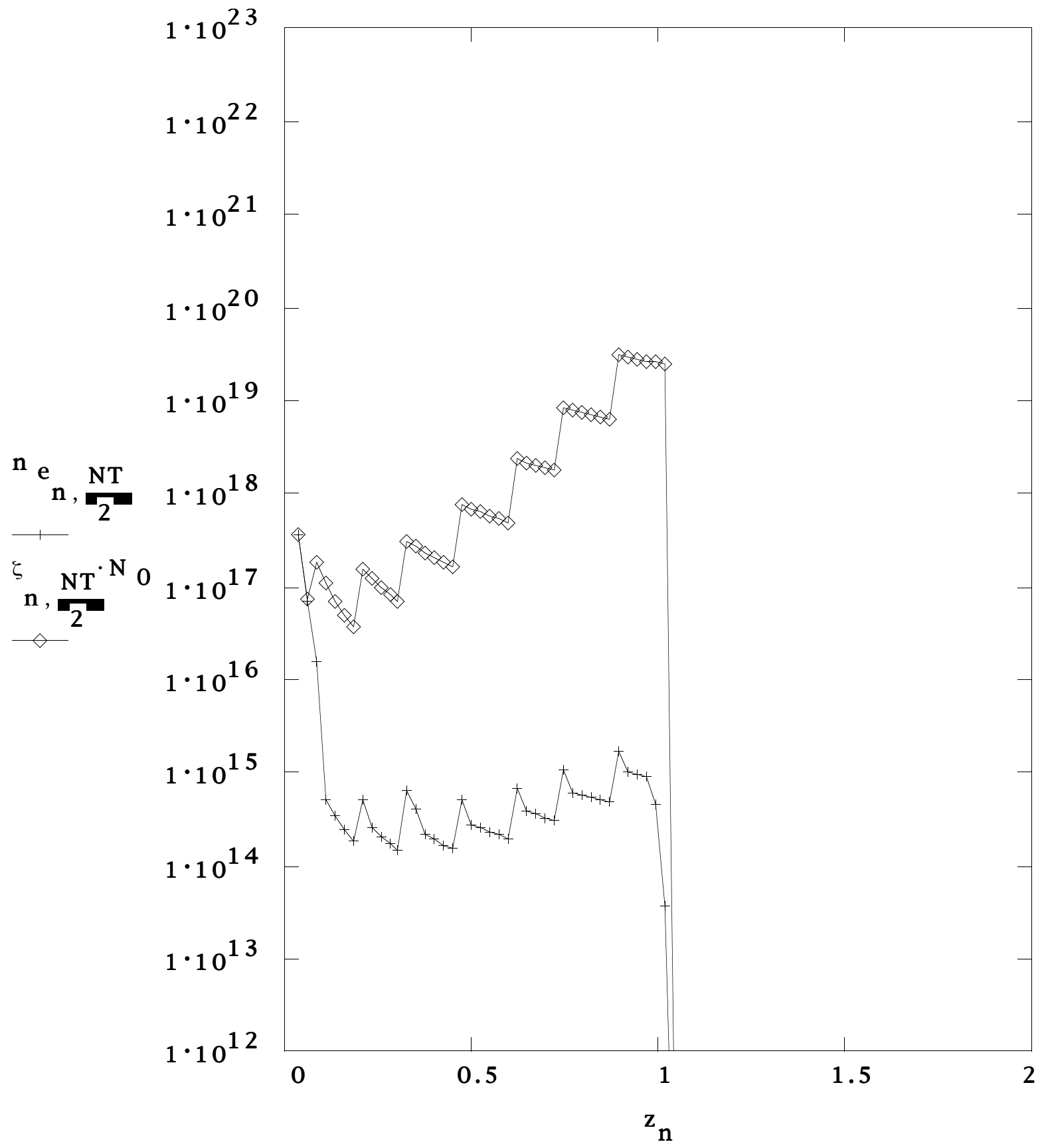

Figure 7: Radial profiles of neutral and plasma densities Densities in $\mathrm{cm}^{\wedge}-3$, distance in $\mathrm{cm}$, point locations shown, neutral points $=<>$ (upper line), plasma points $=+($ lower line $)$ 


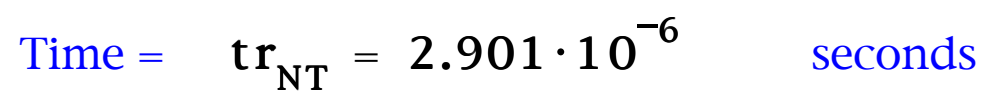

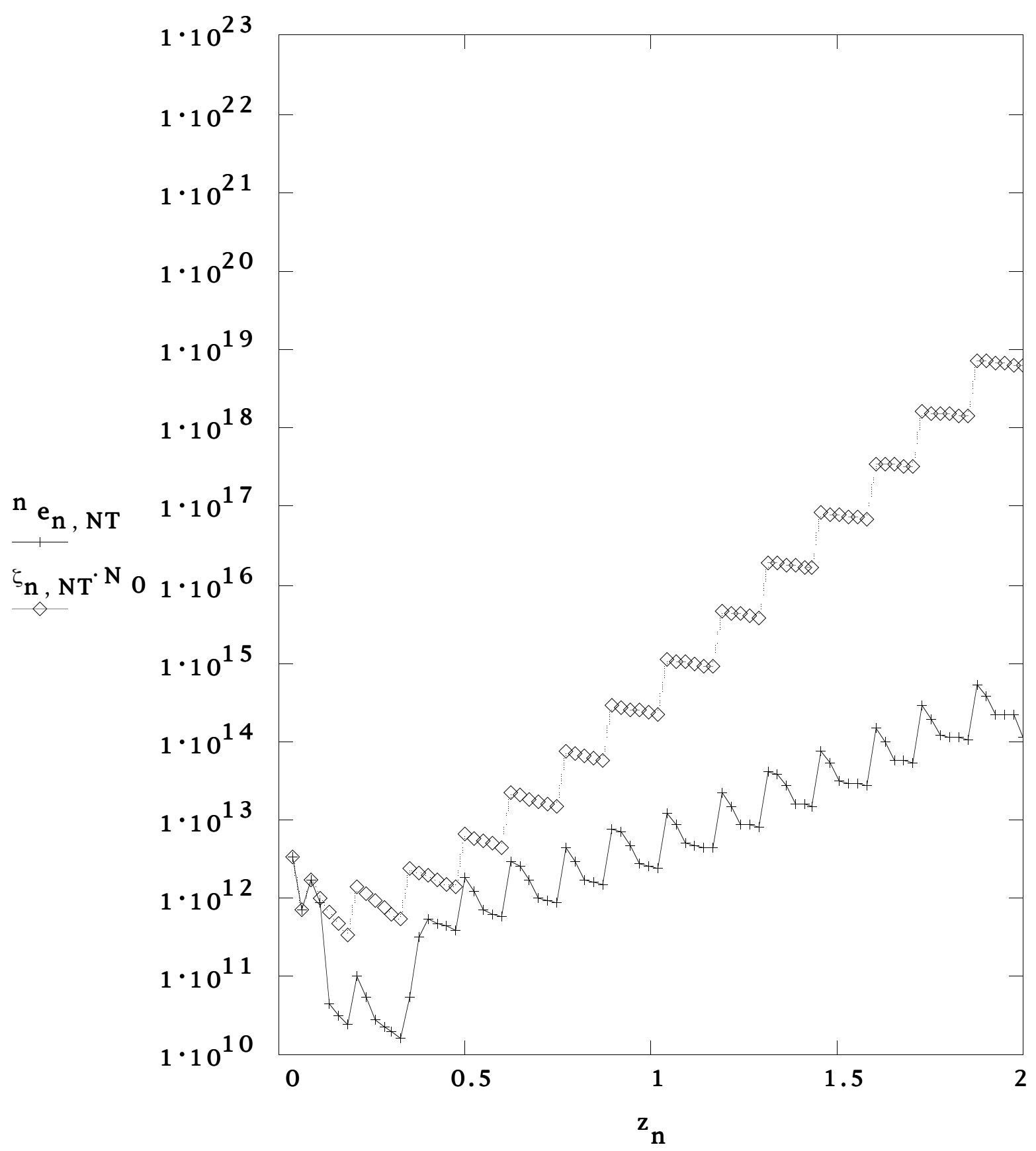

Figure 8: Radial profiles of neutral and plasma densities Densities in $\mathrm{cm}^{\wedge}-3$, distance in $\mathrm{cm}$, point locations shown, neutral points $=<>$ (upper line), plasma points $=+$ (lower line $)$ 


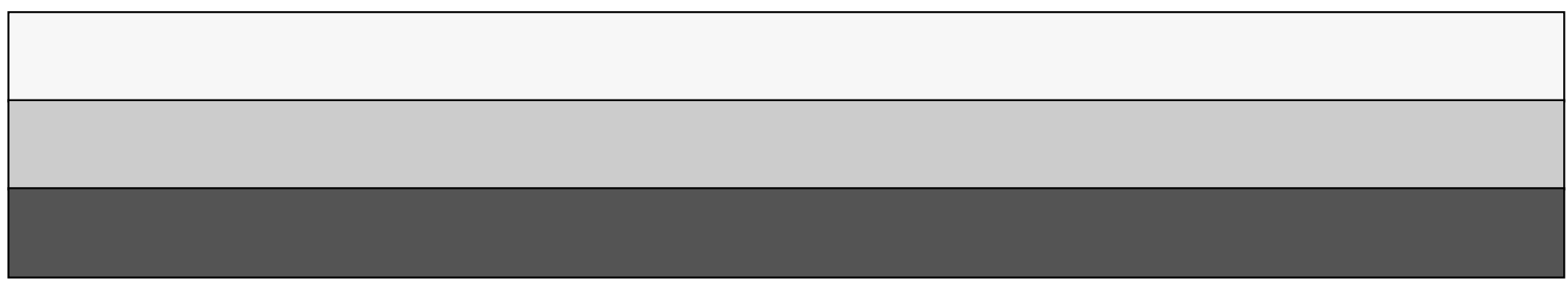

